\title{
Características de tolerância à seca em genótipos de uma coleção nuclear de arroz de terras altas
}

\author{
Thiago Gledson Rios Terra(1), Tarcísio Castro Alves de Barros Leal(2), \\ Paulo Hideo Nakano Rangel ${ }^{(3)}$ e Aluízio Borém de Oliveira ${ }^{(1)}$
}

\begin{abstract}
(1)Universidade Federal de Viçosa, Avenida Peter Henry Rolfs, s/non, Campus Universitário, CEP 36570-900 Viçosa, MG, Brasil. E-mail: tgterra@uft.edu.br, borem@ufv.br (2)Universidade Federal do Tocantins, Rua Badejós, Lote 7, Chácaras 69/72, Zona Rural, Caixa Postal 66, CEP 77402-970 Gurupi, TO, Brasil. E-mail: tarcisio@uft.edu.br ${ }^{(3)}$ Embrapa Arroz e Feijão, Rodovia GO-462, Km 12, Zona Rural, Caixa Postal 179, CEP 75375-000 Santo Antônio de Goiás, GO, Brasil. E-mail: paulo.hideo@embrapa.br
\end{abstract}

Resumo - O objetivo deste trabalho foi avaliar características morfofisiológicas relacionadas à tolerância ao estresse hídrico em genótipos de uma coleção nuclear temática de arroz de terras altas, bem como identificar os materiais mais tolerantes. Foram avaliados 100 genótipos de arroz de terras altas em experimentos conduzidos com e sem estresse por deficiência hídrica. Os genótipos tenderam a aumentar o número de dias para o florescimento (FLO) e a diminuir a altura (ALT), na condição de estresse, mas manifestaram ampla variação de comportamento. A esterilidade das espiguetas (EST) foi a característica mais importante para a seleção, nas duas condições de cultivo, em razão de sua elevada correlação negativa com a produtividade. O número de perfilhos não diferiu entre os genótipos na condição sem estresse, mas houve diferença significativa sob estresse hídrico. Os genótipos Ligeiro e Canela de Ferro apresentaram baixos índices de susceptibilidade à seca (ISS), mas baixa produtividade em ambas as condições de cultivo; já os materiais Catetão, Jatobá e Arroz do Maranhão apresentaram elevada produtividade sob estresse e valores de ISS relativamente baixos. EST, FLO, ALT e ISS são características morfofisiológicas relacionadas à tolerância à seca, e os genótipos Catetão, Jatobá e Arroz do Maranhão podem constituir boas fontes de genes para tolerância à seca em programas de melhoramento.

Termos para indexação: Oryza sativa, arroz de sequeiro, estresse abiótico, estresse hídrico, índice de susceptibilidade, variabilidade genética.

\section{Drought tolerance traits in genotypes from an upland rice core collection}

\begin{abstract}
The objective of this work was to evaluate morphophysiological traits related to drought stress tolerance in genotypes from a thematic upland rice core collection, and to identify the most tolerant materials. One hundred upland rice genotypes were evaluated in experiments carried out with or without drought stress. The genotypes tended to increase the number of days to flowering (FLO) and to decrease plant height (ALT) under stress condition, but showed a great variation in behavior. Spikelet sterility (EST) was the most important trait for selection, in both growing conditions, because of its high negative correlation with yield. The number of tillers did not differ among genotypes, under no stress condition, but there was a significant difference under stress condition. The Ligeiro and Canela de Ferro genotypes showed low drought susceptibility indexes (SID), but low yields in both growing conditions; the Catetão, Jatobá, and Arroz do Maranhão materials had high productivity under stress and relatively low SID values. EST, FLO, ALT, and SID are morphophysiological traits related to drought tolerance, and the Catetão, Jatobá, and Arroz do Maranhão genotypes can be a good source of genes for drought tolerance in breeding programs.
\end{abstract}

Index terms: Oryza sativa, upland rice, abiotic stress, drought stress, susceptibility index, genetic variability.

\section{Introdução}

A maior parte da produção agrícola no Brasil, principalmente a de cereais e oleaginosas, ocorre no verão, quando o suprimento hídrico necessário para o desenvolvimento da cultura provém unicamente das chuvas, no sistema de cultivo em sequeiro. Em muitas regiões do País, notadamente no ecossistema Cerrado, é comum a ocorrência de um período de seca denominado de "veranico", caracterizado pela ausência temporária de chuvas durante a estação chuvosa.

O estresse hídrico afeta diversos processos bioquímicos, fisiológicos e morfológicos nas plantas, e as respostas comumente dependem do genótipo, do 
estádio de desenvolvimento da planta, e da duração e da severidade do estresse (Vidal et al., 2005). Em plantas sob essa condição, há aumento da resistência difusiva ao vapor de água mediante o fechamento dos estômatos, o que reduz a transpiração e o suprimento de $\mathrm{CO}_{2}$, além de diminuir o crescimento celular e aumentar a fotorrespiração (Shinozaki \& Yamaguchi-Shinozaki, 2007). Esses efeitos resultam em perda de vigor, diminuição na altura da planta, aumento na esterilidade do grão de pólen e redução na produtividade (Jongdee et al., 2006; Jagadish et al., 2007; Jin et al., 2013).

Características para tolerância à seca em arroz estão relacionadas à capacidade de uso moderado de água pelas plantas, à redução na área foliar (Nguyen et al., 1997) e à habilidade das raízes em explorar camadas mais profundas do solo (Uga et al., 2013).

A melhoria na estabilidade da produção dos genótipos em ambientes propensos ao deficit hídrico é de grande importância para a cultura do arroz de sequeiro e pode ser feita por meio do melhoramento, pela identificação das características que possam contribuir para a tolerância à seca (Babu et al., 2003). A busca por cultivares de arroz de terras altas tolerantes à seca é reconhecida como a estratégia mais eficiente para aliviar a insegurança alimentar causada pela escassez de água (Huang et al., 2007). Nesse sentido, a avaliação dessa característica em uma coleção nuclear temática para tolerância à seca, que necessariamente apresenta grande variação genética para a característica, tem grande relevância.

O objetivo deste trabalho foi avaliar características morfofisiológicas relacionadas à tolerância ao estresse hídrico em genótipos de uma coleção nuclear temática de arroz de terras altas, bem como identificar os materiais mais tolerantes.

\section{Material e Métodos}

O experimento foi conduzido de junho a novembro de 2007, na estação experimental da Universidade Federal do Tocantins, no Campus Universitário de Gurupi. Nesse período, as temperaturas máxima e mínima ficaram em torno de 35 e $15^{\circ} \mathrm{C}$, respectivamente; a umidade relativa do ar foi de aproximadamente $12 \%$; e houve ausência de chuvas de junho a setembro, e chuvas esporádicas em outubro e novembro.

Foram avaliados 86 acessos componentes da coleção nuclear temática de arroz para tolerância à seca (Pessoa-Filho et al., 2007), além de 14 cultivares e linhagens que serviram de testemunhas. Planejou-se o uso do delineamento experimental de látice triplo $10 \times 10$. No entanto, em razão da perda do tratamento 82 (Caiana Grande) e da baixa eficiência do látice, optouse pelo uso do delineamento de blocos ao acaso, que é uma alternativa ao delineamento em látice (Centeno, 2001), com três repetições. As parcelas foram formadas por quatro linhas de 3,0 $\mathrm{m}$ de comprimento, espaçadas em $0,35 \mathrm{~m}$. A densidade de semeadura utilizada foi de 80 sementes por metro.

A adubação de plantio foi feita com formulação N-P-K mais $\mathrm{Zn}$, nas proporções de $12,90,48$ e $20 \mathrm{~kg} \mathrm{ha}^{-1}$ de $\mathrm{N}, \mathrm{P}_{2} \mathrm{O}_{5}, \mathrm{~K}_{2} \mathrm{O}$ e $\mathrm{ZnSO}_{4}$, respectivamente. A semeadura foi realizada sob plantio convencional. As adubações de cobertura foram feitas a lanço, aos 20 e 90 dias após a emergência (DAE) das plantas, tendo-se utilizado $45 \mathrm{~kg} \mathrm{ha}^{-1} \mathrm{de} \mathrm{N}$, na forma de ureia, em cada aplicação.

A área experimental foi irrigada sem distinção entre os tratamentos, até aos 35 DAE, com uso de conjunto autopropelido formado por carrinho com barras laterais dotadas de válvulas reguladoras de pressão, na saída dos bicos dissipadores. Após esse período, o tratamento sob estresse hídrico recebeu metade da lâmina de água fornecida no tratamento sem estresse, até ao final do ciclo das plantas. Para a condição sem estresse, considerou-se uma tensão de água no solo de até $25 \mathrm{kPa}$ (Stone et al., 1986). O turno de rega foi ajustado conforme a evapotranspiração da cultura, avaliada com auxílio de tensiômetros instalados com cápsula porosa a $0,15 \mathrm{~m}$ de profundidade no solo.

Aos 25 dias após o início do estresse, iniciaram-se as coletas de dados para avaliação de: temperatura do dossel foliar (TDF); floração média (FLO), equivalente ao número de DAE até que $50 \%$ dos perfilhos estivessem floridos; altura de planta (ALT), medida em centímetros, em dez plantas por parcela, do solo à extremidade do perfilho mais alto; número de perfilhos (PER) e panículas por planta (PAN), medido pela contagem em 1,0 $\mathrm{m}$ de sulco, na época de colheita, e transformado para metro quadrado; percentagem de esterilidade de espiguetas (EST); peso de 100 grãos (P100), em gramas; índice de susceptibilidade à seca (ISS), descrito adiante; e produtividade média de grãos, estimada a partir da colheita das duas linhas centrais das parcelas, tendo-se excluído $0,5 \mathrm{~m}$ das extremidades.

A TDF foi avaliada com cinco leituras e duas repetições por leitura, o que totalizou dez estimativas de temperatura em cada parcela. As leituras foram feitas com termômetro a laser Raynger ST (Raytek 
Corporation, Santa Cruz, CA, USA) colocado a $10 \mathrm{~cm}$ do dossel foliar, imediatamente antes da irrigação, nos horários mais quentes do dia, entre $12 \mathrm{~h} 30$ e $14 \mathrm{~h} 00$; ou seja, teoricamente, a temperatura foi medida com o nível mais elevado de tensão de água no solo, tanto no tratamento com estresse quanto no sem.

A EST foi obtida pela contagem dos grãos cheios e vazios em uma amostra de dez panículas maduras por parcela experimental. Para as análises de variância, esses dados foram transformados pela expressão $(\mathrm{x}+1)^{0,5}$.

O ISS foi calculado de acordo com Fischer \& Maurer

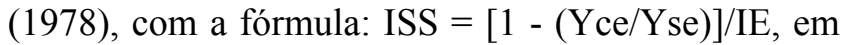
que: Yce é a produtividade do genótipo sob estresse; Yse é a produtividade sem estresse; e IE é a intensidade do estresse aplicado. A intensidade do estresse foi considerada como: IE = 1 - (Pce/Pse), em que Pce é a produtividade média do ensaio com estresse, e Pse é a produtividade média do ensaio sem estresse.

As análises estatísticas foram feitas com o programa Genes (Universidade Federal de Viçosa, Viçosa, MG, Brasil). O teste de agrupamento de médias utilizado foi o de Scott-Knott, para os genótipos, e o de Tukey, para os ambientes. Além disso, avaliou-se a estimativa de correlações entre as características avaliadas nos dois ambientes.

\section{Resultados e Discussão}

$\mathrm{Na}$ análise de variância conjunta (Tabela 1), a única característica que não sofreu efeito dos tratamentos foi a temperatura do dossel foliar. A análise evidenciou ainda que os genótipos foram bastante sensíveis às diferentes condições de cultivo avaliadas.
Apesar do baixo coeficiente de variação, os genótipos não diferiram significativamente entre si, quanto à característica TDF (Tabela 2). Entretanto, houve diferenças significativas entre os ambientes de cultivo, nas avaliações individuais para cada cultivar, tendo-se observado tendência de aumento de temperatura na condição estressada, com aumento médio de $4,04^{\circ} \mathrm{C}$ na TDF. A variedade Palha Murcha apresentou o maior aumento, de $6,99^{\circ} \mathrm{C}$. A redução na abertura estomática é, possivelmente, a responsável pelo aumento na TDF, por reduzir o fluxo transpiratório, que é o principal meio de dissipação térmica das plantas. Jagadish et al. (2007) ressaltaram que TDF elevadas podem aumentar a esterilidade das espiguetas. Contudo, essa variável não se correlacionou com nenhuma outra, na avaliação entre os dois ambientes (Tabela 3).

Quanto à variável FLO, verificaram-se diferenças altamente significativas entre as duas condições de cultivo (Tabela 1), mas com grande amplitude de variação nos resultados (Tabela 2). Por se tratar de uma coleção nuclear, que, por definição, abarca grande diversidade genética, esse resultado era esperado. Assim, foram observados genótipos de ciclo muito curto, como o 64 Dias (67 e 76 DAE nas condições sem e com estresse de seca, respectivamente), até muito tardios, como o Tapuripa (156 e 160 DAE, nas duas condições, respectivamente). O período de florescimento pode ser usado como alternativa,no manejo de variedades para escape à seca. Além disso, há relatos de que plantas sob condições moderadas de estresse hídrico aceleram o florescimento e o enchimento dos grãos, antes que ele se torne muito severo (Xu et al., 2005). No entanto, no presente trabalho, houve aumento médio de 17 dias para FLO, na

Tabela 1. Análise de variância conjunta nos ambientes sem e com estresse hídrico, para as características: temperatura do dossel foliar (TDF), número de dias da emergência à floração média (FLO), altura de planta (ALT), produtividade de grãos (PRO), percentagem de esterilidade de espiguetas (EST), peso de 100 grãos (P100), e número de perfilhos (PER) e panículas (PAN) por metro.

\begin{tabular}{|c|c|c|c|c|c|c|c|c|c|}
\hline $\mathrm{FV}$ & GL & TDF & FLO & ALT & PRO & $\mathrm{EST}^{(1)}$ & $\mathrm{P} 100$ & PER & PAN \\
\hline Blocos & 2 & 19,54 & 259,29 & 577,85 & $45.104,57$ & 1,29 & 0,20 & $41.895,13$ & $32.177,75$ \\
\hline Tratamentos $(\mathrm{T})$ & 98 & $3,26^{\mathrm{ns}}$ & $1.721,4 * *$ & $1.473,10 * *$ & $42.489,88^{* *}$ & $6,02 * *$ & $1,27 * *$ & $4.568,10 * *$ & $3.095,48 * *$ \\
\hline Ambientes (A) & 1 & $2.429,04 * *$ & $46.862,25 * *$ & $57.763,43^{* *}$ & $10.594 .160,90 * *$ & $816,01 * *$ & $27,24 * *$ & $421,35^{* *}$ & $757.295,96^{* *}$ \\
\hline T vs. A & 98 & $3,29^{\text {ns }}$ & $100,46^{* *}$ & $107,13 *$ & $18.105,91 * *$ & $1,23 *$ & $0,14 * *$ & $1.873,03^{\mathrm{ns}}$ & $1.376,46^{\mathrm{ns}}$ \\
\hline Resíduo & 394 & 3,04 & 31,24 & 77,26 & $8.906,33$ & 0,81 & 0,04 & $2.110,96$ & $1.706,21$ \\
\hline Média & & 36,94 & 121,39 & 108,48 & 259,90 & 6,43 & 2,64 & 226,88 & 179,17 \\
\hline $\mathrm{CV}(\%)$ & & 4,73 & 4,60 & 8,13 & 35,48 & 14,00 & 7,20 & 20,29 & 22,91 \\
\hline
\end{tabular}

${ }^{(1)}$ Dados transformados para $(\mathrm{x}+1)^{0,5}$. ${ }^{\text {ns }}$ Não significativo. * $\mathrm{e} * *$ Significativo a 5 e $1 \%$ de probabilidade, respectivamente. 
condição de estresse (Tabela 2). Essa variação entre os ambientes de cultivo pode ser atribuída à necessidade do genótipo de priorizar a formação de raízes, ou de outras partes vegetativas da planta, sob a condição de estresse (Uga et al., 2013), no intuito de aumentar a produção de fotoassimilados.

Tabela 2. Médias nas condições sem (SE) e com estresse (CE), das variáveis: temperatura do dossel foliar (TDF), número de dias da emergência à floração média (FLO), altura de planta (ALT), percentagem de esterilidade de espiguetas (EST), peso de 100 grãos (P100), produtividade de grãos (PRO), número de perfilhos (PER) e panículas (PAN) por metro, e índice de suscetibilidade à seca (ISS) dos 86 acessos que compõem a coleção nuclear temática para tolerância à seca, e de 14 cultivares ou linhagens de arroz de sequeiro (Oryza sativa) ${ }^{(1)}$.

\begin{tabular}{|c|c|c|c|c|c|c|c|c|c|c|c|c|c|c|c|c|c|}
\hline \multirow[t]{2}{*}{ Lista de genótipos } & \multicolumn{2}{|c|}{$\mathrm{TDF}\left({ }^{\circ} \mathrm{C}\right)$} & \multicolumn{2}{|c|}{ FLO } & \multicolumn{2}{|c|}{$\operatorname{ALT}(\mathrm{cm})$} & \multicolumn{2}{|c|}{ EST $(\%)$} & \multicolumn{2}{|c|}{$\mathrm{P} 100(\mathrm{~g})$} & \multicolumn{2}{|c|}{ PRO $\left(\right.$ g parcela $\left.^{-1}\right)$} & \multicolumn{2}{|c|}{ PER } & \multicolumn{2}{|c|}{ PAN } & \multirow[t]{2}{*}{ ISS } \\
\hline & SE & $\mathrm{CE}$ & SE & $\mathrm{CE}$ & SE & $\mathrm{CE}$ & SE & $\mathrm{CE}$ & SE & $\mathrm{CE}$ & SE & $\mathrm{CE}$ & SE & $\mathrm{CE}$ & SE & $\mathrm{CE}$ & \\
\hline Cateto Seda & $34,6 \mathrm{Ba}$ & $39,4 \mathrm{Aa}$ & $92 \mathrm{Bh}$ & $117 \mathrm{Ae}$ & $115 \mathrm{Ad}$ & $96 \mathrm{Bd}$ & $34 \mathrm{Bc}$ & $73 \mathrm{Aa}$ & $3,49 \mathrm{Ac}$ & $2,53 \mathrm{Bb}$ & $171 \mathrm{Ac}$ & $12,9 \mathrm{Bc}$ & $306 \mathrm{Aa}$ & $182 \mathrm{Bb}$ & $255 \mathrm{Aa}$ & $132 \mathrm{Bb}$ & $1,37 \mathrm{a}$ \\
\hline Maruim & $35,7 \mathrm{Ba}$ & $39,8 \mathrm{Aa}$ & $123 \mathrm{Bd}$ & $137 \mathrm{Ac}$ & $124 \mathrm{Ac}$ & $99 \mathrm{Bc}$ & $33 \mathrm{Bc}$ & $57 \mathrm{Ab}$ & $2,18 \mathrm{Ai}$ & $2,06 \mathrm{Ac}$ & $245 \mathrm{Ab}$ & $38,6 \mathrm{Bc}$ & $295 \mathrm{Aa}$ & $185 \mathrm{Bb}$ & 241Aa & $134 \mathrm{Bb}$ & $1,24 \mathrm{a}$ \\
\hline Cajueiro Liso & $35,7 \mathrm{Ba}$ & $39,2 \mathrm{Aa}$ & $118 \mathrm{Bd}$ & $133 \mathrm{Ac}$ & $135 \mathrm{Ac}$ & $107 \mathrm{Bc}$ & $11 \mathrm{Be}$ & $31 \mathrm{Ac}$ & $3,02 \mathrm{Ae}$ & $2,6 \mathrm{Bb}$ & $384 \mathrm{Aa}$ & $183 \mathrm{Ba}$ & $260 \mathrm{Aa}$ & $148 \mathrm{Bb}$ & 209Aa & $118 \mathrm{Bb}$ & $0,77 \mathrm{~b}$ \\
\hline Enche Tulha & $34,1 \mathrm{Ba}$ & $40 \mathrm{Aa}$ & $121 \mathrm{Bd}$ & $138 \mathrm{Ac}$ & $151 \mathrm{Aa}$ & $113 \mathrm{Bc}$ & $37 \mathrm{Ac}$ & $56 \mathrm{Ab}$ & $2,99 \mathrm{Ae}$ & $2,64 \mathrm{Bb}$ & $326 \mathrm{Aa}$ & $93,3 \mathrm{Bc}$ & $253 \mathrm{Aa}$ & $138 \mathrm{Bb}$ & $192 \mathrm{Aa}$ & $102 \mathrm{Bb}$ & $1,05 \mathrm{a}$ \\
\hline Formosa & $34,6 \mathrm{Ba}$ & 39Aa & $111 \mathrm{Bf}$ & $128 \mathrm{Ad}$ & $87 \mathrm{Ae}$ & $62 \mathrm{Be}$ & $6 \mathrm{Be}$ & $52 \mathrm{Ab}$ & 2,3Ai & $2,02 \mathrm{Ac}$ & $343 \mathrm{Aa}$ & $41,4 \mathrm{Bc}$ & $293 \mathrm{Aa}$ & $190 \mathrm{Bb}$ & $240 \mathrm{Aa}$ & $153 \mathrm{Ba}$ &, $30 \mathrm{a}$ \\
\hline Arroz Maranhão & $34,8 \mathrm{Ba}$ & $39,3 \mathrm{Aa}$ & $121 \mathrm{Bd}$ & $140 \mathrm{Ac}$ & $121 \mathrm{Ac}$ & $104 \mathrm{Bc}$ & $25 \mathrm{Bd}$ & $55 \mathrm{Ab}$ & 2,89 Af & $2,21 \mathrm{Bc}$ & $239 \mathrm{Ab}$ & $59,5 \mathrm{Bc}$ & $261 \mathrm{Aa}$ & $151 \mathrm{Bb}$ & 199Aa & $121 \mathrm{Bb}$ & $1,11 \mathrm{a}$ \\
\hline Paulista Dourado & $35,5 \mathrm{Ba}$ & $38,7 \mathrm{Aa}$ & $85 \mathrm{Bi}$ & $99 \mathrm{Ag}$ & $100 \mathrm{Ae}$ & 90Ad & $33 \mathrm{Bc}$ & $55 \mathrm{Ab}$ & $3,88 \mathrm{Aa}$ & $3 \mathrm{Ba}$ & $220 \mathrm{Ab}$ & $51 \mathrm{Bc}$ & $278 \mathrm{Aa}$ & $238 \mathrm{Aa}$ & $220 \mathrm{Aa}$ & $172 \mathrm{Aa}$ & $1,13 \mathrm{a}$ \\
\hline Meruim Ligeiro & $35 \mathrm{Ba}$ & $39,8 \mathrm{Aa}$ & $85 \mathrm{Bi}$ & $113 \mathrm{Af}$ & $97 \mathrm{Ae}$ & 89Ad & $27 \mathrm{Bd}$ & $54 \mathrm{Ab}$ & $3,02 \mathrm{Ae}$ & $2,24 \mathrm{Bc}$ & $212 \mathrm{Ab}$ & $67,6 \mathrm{Bc}$ & $244 \mathrm{Aa}$ & $252 \mathrm{Aa}$ & 196Aa & $176 \mathrm{Aa}$ & $1,00 \mathrm{~b}$ \\
\hline Maranhão Verdadei & $35,3 \mathrm{Ba}$ & $38,9 \mathrm{Aa}$ & $118 \mathrm{Bd}$ & $137 \mathrm{Ac}$ & $128 \mathrm{Ac}$ & $113 \mathrm{Bc}$ & $19 \mathrm{Be}$ & $46 \mathrm{Ab}$ & $2,62 \mathrm{Ag}$ & $2,39 \mathrm{Ab}$ & 291Aa & $128 \mathrm{Bb}$ & $248 \mathrm{Aa}$ & $194 \mathrm{Ab}$ & 198Aa & $152 \mathrm{Aa}$ & $0,83 \mathrm{~b}$ \\
\hline Amarelão Ligeiro & $34,7 \mathrm{Ba}$ & $38,3 \mathrm{Aa}$ & 83Bi & $100 \mathrm{Ag}$ & $103 \mathrm{Ad}$ & $84 \mathrm{Bd}$ & $27 \mathrm{Bd}$ & $63 \mathrm{Aa}$ & $3,7 \mathrm{Ab}$ & $2,94 \mathrm{Ba}$ & $255 \mathrm{Ab}$ & $38,1 \mathrm{Bc}$ & $310 \mathrm{Aa}$ & $242 \mathrm{Aa}$ & $269 \mathrm{Aa}$ & $164 \mathrm{Ba}$ & $1,26 \mathrm{a}$ \\
\hline Puteca & $34,7 \mathrm{Ba}$ & 39,9Aa & $117 \mathrm{Be}$ & $132 \mathrm{Ad}$ & $121 \mathrm{Ac}$ & $99 \mathrm{Bc}$ & $24 \mathrm{Bd}$ & $60 \mathrm{Aa}$ & $3,51 \mathrm{Ac}$ & $2,54 \mathrm{Bb}$ & $321 \mathrm{Aa}$ & $74,3 \mathrm{Bc}$ & $246 \mathrm{Aa}$ & $174 \mathrm{Ab}$ & $193 \mathrm{Aa}$ & $141 \mathrm{Ab}$ & $1,13 \mathrm{a}$ \\
\hline Comum Crioulo & $35,9 \mathrm{Ba}$ & $40,1 \mathrm{Aa}$ & $87 \mathrm{Bi}$ & $111 \mathrm{Af}$ & 110Ad & $86 \mathrm{Bd}$ & $23 \mathrm{Bd}$ & $72 \mathrm{Aa}$ & $3,48 \mathrm{Ac}$ & $3,02 \mathrm{Ba}$ & $256 \mathrm{Ab}$ & $30,7 \mathrm{Bc}$ & $284 \mathrm{Aa}$ & $199 \mathrm{Bb}$ & $218 \mathrm{Aa}$ & $146 \mathrm{Bb}$ & $1,30 \mathrm{a}$ \\
\hline Pratão Goiano & $35,9 \mathrm{Ba}$ & $39,6 \mathrm{Aa}$ & $85 \mathrm{Bi}$ & $111 \mathrm{Af}$ & 110Ad & $90 \mathrm{Bd}$ & $22 \mathrm{Bd}$ & 69Aa & 3,79Aa & $3,02 \mathrm{Ba}$ & $307 \mathrm{Aa}$ & $73,3 \mathrm{Bc}$ & $251 \mathrm{Aa}$ & $187 \mathrm{Ab}$ & $221 \mathrm{Aa}$ & $145 \mathrm{Bb}$ & $1,12 \mathrm{a}$ \\
\hline Prata Branco & $33,9 \mathrm{Ba}$ & $40,1 \mathrm{Aa}$ & $116 \mathrm{Be}$ & $137 \mathrm{Ac}$ & $131 \mathrm{Ac}$ & $117 \mathrm{Ab}$ & $21 \mathrm{Bd}$ & 39Ac & $2,8 \mathrm{Ag}$ & $2,31 \mathrm{Bc}$ & $346 \mathrm{Aa}$ & $118 \mathrm{Bb}$ & $80 \mathrm{Aa}$ & $167 \mathrm{Bb}$ & $218 \mathrm{Aa}$ & $125 \mathrm{Bb}$ & $0,97 \mathrm{a}$ \\
\hline Palha Murcha & $33,2 \mathrm{Ba}$ & $40,1 \mathrm{Aa}$ & $122 \mathrm{Bd}$ & $138 \mathrm{Ac}$ & $126 \mathrm{Ac}$ & $112 \mathrm{Ac}$ & $14 \mathrm{Be}$ & $41 \mathrm{Ac}$ & $3,54 \mathrm{Ac}$ & $3,18 \mathrm{Ba}$ & $340 \mathrm{Aa}$ & $161 \mathrm{Bb}$ & $251 \mathrm{Aa}$ & $163 \mathrm{Bb}$ & 196Aa & $120 \mathrm{Bb}$ & $0,78 b$ \\
\hline Cano Roxo & $33,7 \mathrm{Ba}$ & $38,8 \mathrm{Aa}$ & $114 \mathrm{Be}$ & $135 \mathrm{Ac}$ & $130 \mathrm{Ac}$ & $107 \mathrm{Bc}$ & $14 \mathrm{Be}$ & $40 \mathrm{Ac}$ & $2,78 \mathrm{Ag}$ & $2,54 \mathrm{Ab}$ & $328 \mathrm{Aa}$ & $87,1 \mathrm{Bc}$ & $248 \mathrm{Aa}$ & $184 \mathrm{Ab}$ & $191 \mathrm{Aa}$ & $129 \mathrm{Ab}$ & $1,08 \mathrm{a}$ \\
\hline Chatão Vermelho & $34,5 \mathrm{Ba}$ & $39,7 \mathrm{Aa}$ & $120 \mathrm{Bd}$ & $139 \mathrm{Ac}$ & $124 \mathrm{Ac}$ & $105 \mathrm{Bc}$ & $19 \mathrm{Be}$ & $39 \mathrm{Ac}$ & $3,66 \mathrm{Ab}$ & $2,74 \mathrm{Bb}$ & $295 \mathrm{Aa}$ & $82,4 \mathrm{Bc}$ & $232 \mathrm{Aa}$ & $158 \mathrm{Ab}$ & 203Aa & $127 \mathrm{Bb}$ & $1,06 \mathrm{a}$ \\
\hline Ligeiro & $34,4 \mathrm{Ba}$ & $36,9 \mathrm{Aa}$ & $95 \mathrm{Bh}$ & $121 \mathrm{Ae}$ & 109Ad & $106 \mathrm{Ac}$ & $44 \mathrm{Ab}$ & $39 \mathrm{Ac}$ & $3,47 \mathrm{Ac}$ & $3,1 \mathrm{Ba}$ & $96,7 \mathrm{Ac}$ & 89Ac & $251 \mathrm{Aa}$ & $188 \mathrm{Ab}$ & $210 \mathrm{Aa}$ & $140 \mathrm{Bb}$ & $0,12 b$ \\
\hline Santo Américo & $34,5 \mathrm{Ba}$ & $38,6 \mathrm{Aa}$ & $121 \mathrm{Bd}$ & $137 \mathrm{Ac}$ & 129Ac & $100 \mathrm{Bc}$ & $21 \mathrm{Bd}$ & $51 \mathrm{Ab}$ & $3,45 \mathrm{Ac}$ & $3,22 \mathrm{Aa}$ & $338 \mathrm{Aa}$ & $94,3 \mathrm{Bc}$ & $311 \mathrm{Aa}$ & $151 \mathrm{Bb}$ & $236 \mathrm{Aa}$ & $113 \mathrm{Bb}$ & $1,06 \mathrm{a}$ \\
\hline Vermelho & $35 \mathrm{Ba}$ & $38,1 \mathrm{Aa}$ & $123 \mathrm{Bd}$ & $134 \mathrm{Ac}$ & $111 \mathrm{Ad}$ & $90 \mathrm{Bd}$ & $26 \mathrm{Bd}$ & $48 \mathrm{Ab}$ & $3,03 \mathrm{Ae}$ & $2,65 \mathrm{Bb}$ & $231 \mathrm{Ab}$ & $81 \mathrm{Bc}$ & $286 \mathrm{Aa}$ & $209 \mathrm{Ba}$ & $245 \mathrm{Aa}$ & $149 \mathrm{Ba}$ & $0,96 \mathrm{a}$ \\
\hline Cacho Duplo & $35,9 \mathrm{Ba}$ & $38,4 \mathrm{Aa}$ & $86 \mathrm{Bi}$ & $103 \mathrm{Ag}$ & $111 \mathrm{Ad}$ & $85 \mathrm{Bd}$ & $38 \mathrm{Bc}$ & $73 \mathrm{Aa}$ & $3,08 \mathrm{Ae}$ & $2,74 \mathrm{Bb}$ & $272 \mathrm{Ab}$ & $47,1 \mathrm{Bc}$ & $267 \mathrm{Aa}$ & $194 \mathrm{Ab}$ & $217 \mathrm{Aa}$ & $138 \mathrm{Bb}$ & $1,22 \mathrm{a}$ \\
\hline Neném & $35,2 \mathrm{Ba}$ & $39,5 \mathrm{Aa}$ & $123 \mathrm{Bd}$ & $144 \mathrm{Ab}$ & $123 \mathrm{Ac}$ & $108 \mathrm{Bc}$ & $18 \mathrm{Be}$ & $38 \mathrm{Ac}$ & $2,63 \mathrm{Ag}$ & $2,46 \mathrm{Ab}$ & $250 \mathrm{Ab}$ & $83,8 \mathrm{Bc}$ & $250 \mathrm{Aa}$ & $185 \mathrm{Ab}$ & $187 \mathrm{Aa}$ & $136 \mathrm{Ab}$ & $0,98 \mathrm{a}$ \\
\hline Amarelão/Douradão & $35,8 \mathrm{Ba}$ & $39,4 \mathrm{Aa}$ & $125 \mathrm{Bd}$ & $141 \mathrm{Ac}$ & $138 \mathrm{Ab}$ & $112 \mathrm{Bc}$ & $15 \mathrm{Be}$ & $38 \mathrm{Ac}$ & $2,86 \mathrm{Af}$ & $2,44 \mathrm{Bb}$ & $278 \mathrm{Aa}$ & $152 \mathrm{Bb}$ & $248 \mathrm{Aa}$ & $172 \mathrm{Ab}$ & $204 \mathrm{Aa}$ & $116 \mathrm{Bb}$ & $0,67 \mathrm{~b}$ \\
\hline Brejeiro/Nenenzinho & $33,7 \mathrm{Ba}$ & $38,9 \mathrm{Aa}$ & $135 \mathrm{Bc}$ & $145 \mathrm{Ab}$ & $156 \mathrm{Aa}$ & $139 \mathrm{Ba}$ & $43 \mathrm{Bb}$ & $76 \mathrm{Aa}$ & 2,39Ah & $2,59 \mathrm{Ab}$ & $235 \mathrm{Ab}$ & $113 \mathrm{Bb}$ & $235 \mathrm{Aa}$ & $161 \mathrm{Ab}$ & $221 \mathrm{Aa}$ & $111 \mathrm{Bb}$ & $0,77 \mathrm{~b}$ \\
\hline Brejeiro & $34,7 \mathrm{Ba}$ & $39,3 \mathrm{Aa}$ & $133 \mathrm{Bc}$ & $149 \mathrm{Ab}$ & $157 \mathrm{Aa}$ & $141 \mathrm{Ba}$ & $37 \mathrm{Bc}$ & 71Aa & $2,45 \mathrm{Ah}$ & $2,59 \mathrm{Ab}$ & $262 \mathrm{Ab}$ & $93,8 \mathrm{Bc}$ & $207 \mathrm{Aa}$ & $143 \mathrm{Ab}$ & $183 \mathrm{Aa}$ & $109 \mathrm{Bb}$ & $0,95 \mathrm{a}$ \\
\hline Quebra Cacho & $34,8 \mathrm{Ba}$ & $38,5 \mathrm{Aa}$ & $124 \mathrm{Bd}$ & $134 \mathrm{Ac}$ & $137 \mathrm{Ab}$ & $116 \mathrm{Bb}$ & $24 \mathrm{Bd}$ & $37 \mathrm{Ac}$ & 2,92Af & $2,69 \mathrm{Ab}$ & $414 \mathrm{Aa}$ & $241 \mathrm{Ba}$ & $244 \mathrm{Aa}$ & $157 \mathrm{Bb}$ & $215 \mathrm{Aa}$ & $123 \mathrm{Bb}$ & $0,62 b$ \\
\hline Bico De Rola & $34,4 \mathrm{Ba}$ & 39,1 & $117 \mathrm{Be}$ & 137 & Ic & 9 & $18 \mathrm{Be}$ & 5 & $3 \mathrm{Ae}$ & $\mathrm{Bb}$ & 37 & $\mathrm{Bb}$ & a & $153 \mathrm{Bb}$ & $170 \mathrm{Aa}$ & $115 \mathrm{Ab}$ & $9 b$ \\
\hline Muruim Branco 1 & $35,1 \mathrm{Ba}$ & Id & $125 \mathrm{Bd}$ & 1 & Ad & 110 & $28 \mathrm{Bd}$ & $\mathrm{b}$ & $\mathrm{Ah}$ & BBd & $\mathrm{Ac}$ & $93,8 \mathrm{Ac}$ & $\mathrm{Aa}$ & $192 \mathrm{Ab}$ & $188 \mathrm{Aa}$ & $147 \mathrm{Ab}$ & $0,71 \mathrm{~b}$ \\
\hline Douradão/Amarelão & $35,4 \mathrm{Ba}$ & $38,2 \mathrm{Aa}$ & $140 \mathrm{Ac}$ & $145 \mathrm{Ab}$ & $152 \mathrm{Aa}$ & $128 \mathrm{Ba}$ & $44 \mathrm{Bb}$ & $67 \mathrm{Aa}$ & 2,31Ai & $2,22 \mathrm{Ac}$ & $229 \mathrm{Ab}$ & $84,8 \mathrm{Bc}$ & $262 \mathrm{Aa}$ & $173 \mathrm{Bb}$ & $231 \mathrm{Aa}$ & $137 \mathrm{Bb}$ & $0,93 \mathrm{~b}$ \\
\hline Iguape Sem Aresta & $35,6 \mathrm{Ba}$ & $38,2 \mathrm{Aa}$ & $121 \mathrm{Bd}$ & $140 \mathrm{Ac}$ & $126 \mathrm{Ac}$ & $109 \mathrm{Bc}$ & $31 \mathrm{Bc}$ & $66 \mathrm{Aa}$ & $2,93 \mathrm{Af}$ & $2,55 \mathrm{Bb}$ & $304 \mathrm{Aa}$ & $63,8 \mathrm{Bc}$ & $226 \mathrm{Aa}$ & $156 \mathrm{Ab}$ & $179 \mathrm{Aa}$ & $125 \mathrm{Ab}$ & $1,17 \mathrm{a}$ \\
\hline Vermelhão & $34,8 \mathrm{Ba}$ & $40,6 \mathrm{Aa}$ & $118 \mathrm{Bd}$ & $142 \mathrm{Ac}$ & $130 \mathrm{Ac}$ & $101 \mathrm{Bc}$ & $17 \mathrm{Be}$ & $62 \mathrm{Aa}$ & $3,53 \mathrm{Ac}$ & $2,61 \mathrm{Bb}$ & $312 \mathrm{Aa}$ & $51 \mathrm{Bc}$ & $243 \mathrm{Aa}$ & $167 \mathrm{Ab}$ & $188 \mathrm{Aa}$ & $120 \mathrm{Ab}$ & $1,23 \mathrm{a}$ \\
\hline Samambaia Amarelo & $35 \mathrm{Ba}$ & $40,1 \mathrm{Aa}$ & $122 \mathrm{Bd}$ & $138 \mathrm{Ac}$ & $124 \mathrm{Ac}$ & $106 \mathrm{Bc}$ & $16 \mathrm{Be}$ & $38 \mathrm{Ac}$ & $2,84 \mathrm{Af}$ & $2,54 \mathrm{Ab}$ & 349Aa & $139 \mathrm{Bb}$ & $257 \mathrm{Aa}$ & $174 \mathrm{Bb}$ & $206 \mathrm{Aa}$ & $125 \mathrm{Bb}$ & $0,89 b$ \\
\hline Samambaia & $35,6 \mathrm{Ba}$ & $39,4 \mathrm{Aa}$ & $123 \mathrm{Bd}$ & $141 \mathrm{Ac}$ & $130 \mathrm{Ac}$ & $105 \mathrm{Bc}$ & $11 \mathrm{Be}$ & $29 \mathrm{Ac}$ & $3,03 \mathrm{Ae}$ & $2,62 \mathrm{Bb}$ & $240 \mathrm{Ab}$ & $131 \mathrm{Bb}$ & $246 \mathrm{Aa}$ & $173 \mathrm{Ab}$ & 207Aa & $136 \mathrm{Bb}$ & $0,67 b$ \\
\hline Paulista & $33,9 \mathrm{Ba}$ & $39,1 \mathrm{Aa}$ & $113 \mathrm{Bf}$ & $131 \mathrm{Ad}$ & $126 \mathrm{Ac}$ & $95 \mathrm{Bd}$ & $16 \mathrm{Be}$ & $36 \mathrm{Ac}$ & $3,53 \mathrm{Ac}$ & $2,69 \mathrm{Bb}$ & $408 \mathrm{Aa}$ & $86,4 \mathrm{Bc}$ & $279 \mathrm{Aa}$ & $184 \mathrm{Bb}$ & 234Aa & $154 \mathrm{Ba}$ & $1,16 \mathrm{a}$ \\
\hline Arroz Carolino & $35,3 \mathrm{Ba}$ & $39,8 \mathrm{Aa}$ & $77 \mathrm{Bj}$ & $118 \mathrm{Ae}$ & $96 \mathrm{Ae}$ & 83Ad & $24 \mathrm{Bd}$ & $64 \mathrm{Aa}$ & 3,34Ad & $2,39 \mathrm{Bb}$ & $448 \mathrm{Aa}$ & $91,4 \mathrm{Bc}$ & $254 \mathrm{Aa}$ & $159 \mathrm{Bb}$ & $226 \mathrm{Aa}$ & $109 \mathrm{Bb}$ & $1,17 \mathrm{a}$ \\
\hline Arroz Catetão & $34,8 \mathrm{Ba}$ & $38,9 \mathrm{Aa}$ & $117 \mathrm{Be}$ & $136 \mathrm{Ac}$ & $122 \mathrm{Ac}$ & $110 \mathrm{Ac}$ & $13 \mathrm{Be}$ & $37 \mathrm{Ac}$ & $3,71 \mathrm{Ab}$ & $3,07 \mathrm{Ba}$ & $324 \mathrm{Aa}$ & $130 \mathrm{Bb}$ & $253 \mathrm{Aa}$ & $143 \mathrm{Bb}$ & $214 \mathrm{Aa}$ & $96 \mathrm{Bb}$ & $0,88 \mathrm{~b}$ \\
\hline Gergelim & $34,4 \mathrm{Ba}$ & $39,7 \mathrm{Aa}$ & $122 \mathrm{Bd}$ & $141 \mathrm{Ac}$ & $123 \mathrm{Ac}$ & 94Bd & $22 \mathrm{Bd}$ & $51 \mathrm{Ab}$ & $2,07 \mathrm{Aj}$ & $1,94 \mathrm{Ad}$ & $266 \mathrm{Ab}$ & $110 \mathrm{Bb}$ & $227 \mathrm{Aa}$ & $182 \mathrm{Ab}$ & $185 \mathrm{Aa}$ & $138 \mathrm{Ab}$ & $0,87 b$ \\
\hline Arroz do Sul & $34,4 \mathrm{Ba}$ & $38,1 \mathrm{Aa}$ & 119Bd & $135 \mathrm{Ac}$ & $134 \mathrm{Ac}$ & $116 \mathrm{Bb}$ & $16 \mathrm{Be}$ & 39Ac & $3,14 \mathrm{Ae}$ & $2,71 \mathrm{Bb}$ & $160 \mathrm{Ac}$ & $120 \mathrm{Ab}$ & $248 \mathrm{Aa}$ & $195 \mathrm{Ab}$ & 201Aa & $153 \mathrm{Aa}$ & $0,37 \mathrm{~b}$ \\
\hline Branco Bico Preto & $35,4 \mathrm{Ba}$ & 39Aа & $112 \mathrm{Bf}$ & $128 \mathrm{Ad}$ & $115 \mathrm{Ad}$ & $97 \mathrm{Bd}$ & $26 \mathrm{Ad}$ & 39Ac & $3,22 \mathrm{Ae}$ & $2,87 \mathrm{Ba}$ & $278 \mathrm{Aa}$ & $161 \mathrm{Bb}$ & $253 \mathrm{Aa}$ & $169 \mathrm{Bb}$ & $200 \mathrm{Aa}$ & $108 \mathrm{Bb}$ & $0,62 b$ \\
\hline Branco 4 Meses & $35,7 \mathrm{Aa}$ & $37,2 \mathrm{Aa}$ & $113 \mathrm{Bf}$ & $124 \mathrm{Ad}$ & $124 \mathrm{Ac}$ & $89 \mathrm{Bd}$ & $19 \mathrm{Be}$ & $51 \mathrm{Ab}$ & $3,28 \mathrm{Ad}$ & $2,69 \mathrm{Bb}$ & 396Aa & $72,4 \mathrm{Bc}$ & $264 \mathrm{Aa}$ & $169 \mathrm{Bb}$ & $210 \mathrm{Aa}$ & $130 \mathrm{Bb}$ & $1,21 \mathrm{a}$ \\
\hline Arroz Cabeludo & $35,3 \mathrm{Ba}$ & $38,7 \mathrm{Aa}$ & $111 \mathrm{Bf}$ & $131 \mathrm{Ad}$ & $126 \mathrm{Ac}$ & 98Bd & $16 \mathrm{Be}$ & $43 \mathrm{Ac}$ & 2,89Af & $2,65 \mathrm{Ab}$ & $317 \mathrm{Aa}$ & $137 \mathrm{Bb}$ & $282 \mathrm{Aa}$ & $189 \mathrm{Bb}$ & $234 \mathrm{Aa}$ & $152 \mathrm{Ba}$ & $0,84 \mathrm{~b}$ \\
\hline Carioca/RC $(2)$ & $35,4 \mathrm{Ba}$ & $39,9 \mathrm{Aa}$ & $99 \mathrm{Bh}$ & $132 \mathrm{Ad}$ & $114 \mathrm{Ad}$ & 94Bd & $26 \mathrm{Bd}$ & $78 \mathrm{Aa}$ & $3,06 \mathrm{Ae}$ & $2,07 \mathrm{Bc}$ & $228 \mathrm{Ab}$ & $59,5 \mathrm{Bc}$ & $297 \mathrm{Aa}$ & $275 \mathrm{Aa}$ & $250 \mathrm{Aa}$ & $207 \mathrm{Aa}$ & $1,09 \mathrm{a}$ \\
\hline Fundo Roxo & $35,6 \mathrm{Ba}$ & 40,9Aa & $110 \mathrm{Bf}$ & $145 \mathrm{Ab}$ & $117 \mathrm{Ac}$ & $96 \mathrm{Bd}$ & $29 \mathrm{Bc}$ & $85 \mathrm{Aa}$ & $2,83 \mathrm{Af}$ & $1,95 \mathrm{Bd}$ & $270 \mathrm{Ab}$ & $31,9 \mathrm{Bc}$ & $317 \mathrm{Aa}$ & $211 \mathrm{Ba}$ & $240 \mathrm{Aa}$ & $167 \mathrm{Ba}$ & $1,30 \mathrm{a}$ \\
\hline 3 Meses Amarelo & $34,5 \mathrm{Ba}$ & $39,2 \mathrm{Aa}$ & $84 \mathrm{Bi}$ & $104 \mathrm{Ag}$ & $104 \mathrm{Ad}$ & $92 \mathrm{Ad}$ & $25 \mathrm{Bd}$ & $67 \mathrm{Aa}$ & $3,83 \mathrm{Aa}$ & $2,88 \mathrm{Ba}$ & $324 \mathrm{Aa}$ & $62,4 \mathrm{Bc}$ & $234 \mathrm{Aa}$ & $188 \mathrm{Ab}$ & $184 \mathrm{Aa}$ & $122 \mathrm{Ab}$ & $1,19 \mathrm{a}$ \\
\hline Branquinho & $34,6 \mathrm{Ba}$ & $39,1 \mathrm{Aa}$ & $82 \mathrm{Bi}$ & $105 \mathrm{Ag}$ & $92 \mathrm{Ae}$ & $90 \mathrm{Ad}$ & $17 \mathrm{Be}$ & $61 \mathrm{Aa}$ & $3,72 \mathrm{Ab}$ & $2,91 \mathrm{Ba}$ & $303 \mathrm{Aa}$ & $60 \mathrm{Bc}$ & 279Aa & $229 \mathrm{Aa}$ & $210 \mathrm{Aa}$ & $194 \mathrm{Aa}$ & $1,18 \mathrm{a}$ \\
\hline
\end{tabular}

Continua... 
Tabela 2. Continuação.

\begin{tabular}{|c|c|c|c|c|c|c|c|c|c|c|c|c|c|c|c|c|c|}
\hline \multirow[t]{2}{*}{ ista de genótipos } & \multicolumn{2}{|c|}{$\operatorname{TDF}\left({ }^{\circ} \mathrm{C}\right)$} & \multicolumn{2}{|c|}{ FLO } & \multicolumn{2}{|c|}{$\operatorname{ALT}(\mathrm{cm})$} & \multicolumn{2}{|c|}{ EST (\%) } & \multicolumn{2}{|c|}{ P100 (g) } & \multicolumn{2}{|c|}{ PRO $\left(\right.$ g parcela- $\left.{ }^{-1}\right)$} & \multicolumn{2}{|l|}{ PER } & \multicolumn{2}{|c|}{ PAN } & \multirow[t]{2}{*}{ ISS } \\
\hline & $\mathrm{SE}$ & $\mathrm{CE}$ & $\mathrm{SE}$ & $\mathrm{CE}$ & $\mathrm{SE}$ & $\mathrm{CE}$ & $\mathrm{SE}$ & $\mathrm{CE}$ & $\mathrm{SE}$ & $\mathrm{CE}$ & $\mathrm{SE}$ & $\mathrm{CE}$ & SE & $\mathrm{CE}$ & SE & $\mathrm{CE}$ & \\
\hline Meses Antig & $33,6 \mathrm{Ba}$ & , $1 \mathrm{Aa}$ & $99 \mathrm{Bf}$ & $27 \mathrm{Ad}$ & $18 \mathrm{Ac}$ & $96 \mathrm{Bd}$ & $20 \mathrm{Bd}$ & $49 \mathrm{Ab}$ & $3,32 \mathrm{Ad}$ & $2,86 \mathrm{Ba}$ & 379Aa & $159 \mathrm{Bb}$ & $265 \mathrm{Aa}$ & $187 \mathrm{Bb}$ & $208 \mathrm{Aa}$ & $125 \mathrm{Bb}$ & $0,86 \mathrm{~b}$ \\
\hline ha & $4 \mathrm{Ba}$ & $2 \mathrm{Aa}$ & $1 \mathrm{Bg}$ & Ac & $5 \mathrm{Ad}$ & & & Aa & $2,66 \mathrm{Ag}$ & & $6 \mathrm{Ab}$ & $77,6 \mathrm{Bc}$ & $2 \mathrm{Aa}$ & $\mathrm{Aa}$ & $17 \mathrm{Aa}$ & $9 \mathrm{Aa}$ & $5 \mathrm{a}$ \\
\hline Irol & $\mathrm{Ba}$ & $\mathrm{Aa}$ & $8 \mathrm{Bd}$ & Ac & Ic & & & & 2,21Ai & & $\mathrm{Ab}$ & & $6 \mathrm{Aa}$ & & & $9 \mathrm{Ab}$ & $0,67 \mathrm{~b}$ \\
\hline rroz do Maranhão & $33,8 \mathrm{Ba}$ & $8,1 \mathrm{Aa}$ & $114 \mathrm{Be}$ & $128 \mathrm{Ad}$ & $19 \mathrm{Ac}$ & 97Bd & 1Bd & $45 \mathrm{Ab}$ & $3,16 \mathrm{Ae}$ & $2,84 \mathrm{Ba}$ & $365 \mathrm{Aa}$ & $219 \mathrm{Ba}$ & $37 \mathrm{Aa}$ & $225 \mathrm{Aa}$ & 79Aa & $80 \mathrm{Aa}$ & $0,59 b$ \\
\hline roz Roxo & i $34,9 \mathrm{Ba}$ & $38,3 \mathrm{Aa}$ & 97Bh & 127Ad & $99 \mathrm{Ae}$ & & e & & $9 \mathrm{Ae}$ & $2,32 \mathrm{Bc}$ & $279 \mathrm{Aa}$ & $89,3 \mathrm{Bc}$ & $59 \mathrm{Aa}$ & $198 \mathrm{Bb}$ & 76Aa & $42 \mathrm{Bb}$ & 0a \\
\hline 10 & $35,6 \mathrm{Ba}$ & $40 \mathrm{Aa}$ & $110 \mathrm{Bf}$ & $135 \mathrm{Ac}$ & 116Ad & $9 \mathrm{Bd}$ & $23 \mathrm{Bd}$ & $2 \mathrm{Ab}$ & 3,3Ad & $2,82 \mathrm{Ba}$ & $305 \mathrm{Aa}$ & $85,7 \mathrm{Bc}$ & $272 \mathrm{Aa}$ & $155 \mathrm{Bb}$ & 209Aa & 117Bb & $1,06 \mathrm{a}$ \\
\hline & $35,1 \mathrm{Ba}$ & $38,4 \mathrm{Aa}$ & $122 \mathrm{Bd}$ & $133 \mathrm{Ad}$ & $33 \mathrm{Ac}$ & $0 \mathrm{Bc}$ & $\mathrm{Bd}$ & & 9Af & $2,75 \mathrm{Ab}$ & $336 \mathrm{Aa}$ & 6Ba & $2 \mathrm{Aa}$ & $7 \mathrm{Bb}$ & $4 \mathrm{Aa}$ & BBb & $0,66 \mathrm{~b}$ \\
\hline $\mathrm{roz}$ & $34,6 \mathrm{Ba}$ & $9,7 \mathrm{Aa}$ & $85 \mathrm{Bi}$ & $125 \mathrm{Ad}$ & $97 \mathrm{Ae}$ & 95Ad & $37 \mathrm{Ac}$ & $54 \mathrm{Ab}$ & $3,32 \mathrm{Ad}$ & $2,66 \mathrm{Bb}$ & $233 \mathrm{Ab}$ & $69,8 \mathrm{Bc}$ & $277 \mathrm{Aa}$ & $173 \mathrm{Bb}$ & $235 \mathrm{Aa}$ & $120 \mathrm{Bb}$ & $1,03 \mathrm{a}$ \\
\hline ito & $1,4 \mathrm{Ba}$ &, $2 \mathrm{Aa}$ & $123 \mathrm{Bd}$ & $135 \mathrm{Ac}$ & $117 \mathrm{Ac}$ & $99 \mathrm{Bc}$ & $11 \mathrm{Be}$ & Ac & $2,84 \mathrm{Af}$ & $2,65 \mathrm{Ab}$ & $289 \mathrm{Aa}$ & $180 \mathrm{Ba}$ & $54 \mathrm{Aa}$ & $173 \mathrm{Bb}$ & $220 \mathrm{Aa}$ & $131 \mathrm{Bb}$ & $0,56 \mathrm{~b}$ \\
\hline & $1 \mathrm{Ba}$ &, $1 \mathrm{Aa}$ & $85 \mathrm{Bi}$ & $102 \mathrm{Ag}$ & $91 \mathrm{Ae}$ & Ad & $32 \mathrm{Bc}$ & $\mathrm{Aa}$ & $3,44 \mathrm{Ac}$ & $2,59 \mathrm{Bb}$ & $162 \mathrm{Ac}$ & $73,8 \mathrm{Ac}$ & $\mathrm{Aa}$ & $138 \mathrm{Bb}$ & $1 \mathrm{Aa}$ & $4 \mathrm{DO}$ & $0,8 \mathrm{~b}$ \\
\hline tetão & $34,3 \mathrm{Ba}$ & $38 \mathrm{Aa}$ & $119 \mathrm{Bd}$ & $132 \mathrm{Ad}$ & $129 \mathrm{Ac}$ & $119 \mathrm{Ab}$ & $16 \mathrm{Ae}$ & $25 \mathrm{Ac}$ & $3,33 \mathrm{Ad}$ & $3,03 \mathrm{Aa}$ & $415 \mathrm{Aa}$ & $51 \mathrm{Ba}$ & $88 \mathrm{Aa}$ & $198 \mathrm{Ab}$ & $94 \mathrm{Aa}$ & $157 \mathrm{Aa}$ & $0,58 \mathrm{~b}$ \\
\hline ine & $35,2 \mathrm{Ba}$ & $40,1 \mathrm{Aa}$ & $95 \mathrm{Bh}$ & $144 \mathrm{Ab}$ & $113 \mathrm{Ad}$ & 106 & $34 \mathrm{Bc}$ & & Af & $2,16 \mathrm{Bc}$ & $99,5 \mathrm{Ac}$ & & $\mathrm{Aa}$ & $213 \mathrm{Aa}$ & & $175 \mathrm{Aa}$ & $0,36 \mathrm{~b}$ \\
\hline gul & $35,3 \mathrm{Ba}$ & $39,9 \mathrm{Aa}$ & $117 \mathrm{Be}$ & $138 \mathrm{Ac}$ & $117 \mathrm{Ac}$ & $100 \mathrm{Bc}$ & $33 \mathrm{Bc}$ & $72 \mathrm{Aa}$ & $2,66 \mathrm{Ag}$ & $2,34 \mathrm{Bc}$ & $193 \mathrm{Ab}$ & $76,7 \mathrm{Bc}$ & $4 \mathrm{Aa}$ & $189 \mathrm{Ab}$ & 209Aa & $152 \mathrm{Aa}$ & $0,89 \mathrm{~b}$ \\
\hline talà & $5 \mathrm{Ba}$ &, $8 \mathrm{Aa}$ & $94 \mathrm{Bh}$ & $119 \mathrm{Ae}$ & 03Ad & & 26 & & $\mathrm{Ag}$ & $\mathrm{Bc}$ & & & $\mathrm{Aa}$ & & Aa & & $4 \mathrm{a}$ \\
\hline Irre & $34,6 \mathrm{Ba}$ & 8,3Aa & $134 \mathrm{Bc}$ & $145 \mathrm{Ab}$ & $124 \mathrm{Ac}$ & $107 \mathrm{Bc}$ & 21Bd & $\mathrm{b}$ & $2,49 \mathrm{Ah}$ & $2,21 \mathrm{Ac}$ & $\mathrm{Aa}$ & $\mathrm{Bb}$ & $\mathrm{Aa}$ & $222 \mathrm{Ba}$ & Aa & Ва & $0,7 \mathrm{~b}$ \\
\hline aks & $1 \mathrm{Ba}$ & $9,4 \mathrm{Aa}$ & $124 \mathrm{Bd}$ & $135 \mathrm{Ac}$ & $128 \mathrm{Ac}$ & $112 \mathrm{Bc}$ & $17 \mathrm{Be}$ & Ac & $2,98 \mathrm{Af}$ & $2,54 \mathrm{Bb}$ & $308 \mathrm{Aa}$ & $166 \mathrm{Bb}$ & $193 \mathrm{Aa}$ & $174 \mathrm{Ab}$ & $163 \mathrm{Aa}$ & 149Aa & $0,68 \mathrm{~b}$ \\
\hline $\mathrm{CD}$ & 7Ba & Aa & $82 \mathrm{Bi}$ & 96Ag & 81 & & 32 & la & $3,95 \mathrm{Aa}$ & $2,89 \mathrm{Ba}$ & 339Aa & $\mathrm{Bc}$ & $\mathrm{Aa}$ & $\mathrm{aa}$ & Aa & a & $6 a$ \\
\hline co & 3Ba & ,2Aa & $84 \mathrm{Bi}$ & $13 \mathrm{Af}$ & 08Ad & & Bc & & $\mathrm{Ae}$ & $3 b$ & $\mathrm{Aa}$ & $\mathrm{Bc}$ & $\mathrm{Aa}$ & $\mathrm{bb}$ & & & $9 \mathrm{a}$ \\
\hline a4 & $35,4 \mathrm{Ba}$ & $40,7 \mathrm{Aa}$ & 109Bf & $135 \mathrm{Ac}$ & $114 \mathrm{Ad}$ & & $37 \mathrm{Bc}$ & la & $2,37 \mathrm{Ah}$ & $2,05 \mathrm{Bc}$ & $251 \mathrm{Ab}$ & $53,6 \mathrm{Bc}$ & $\mathrm{Aa}$ & $229 \mathrm{Ba}$ & 25 & 3a & $1,16 \mathrm{a}$ \\
\hline gull & $34,6 \mathrm{Ba}$ & $9,2 \mathrm{Aa}$ & $150 \mathrm{Ab}$ & $155 \mathrm{Aa}$ & $137 \mathrm{Ab}$ & $117 \mathrm{Bb}$ & $43 \mathrm{Bb}$ & $\mathrm{la}$ & $1,74 \mathrm{Ak}$ & $1,43 \mathrm{Bd}$ & $168 \mathrm{Ac}$ & $1,9 \mathrm{Bc}$ & $239 \mathrm{Aa}$ & $210 \mathrm{Aa}$ & $\mathrm{Aa}$ & $\mathrm{Ab}$ & $1,46 \mathrm{a}$ \\
\hline uru & ,3Ba & 9,4Aa & $125 \mathrm{Bd}$ & $43 \mathrm{Ab}$ & $21 \mathrm{Ac}$ & $A b$ & 30 & c & $\mathrm{Ai}$ & $2,23 \mathrm{Ac}$ & $250 \mathrm{Ab}$ & 7Bb & Aa & $197 \mathrm{Bb}$ & Aa & $\mathrm{Bb}$ & 0 , \\
\hline bró & $35,4 \mathrm{Ba}$ & $9,2 \mathrm{Aa}$ & $136 \mathrm{Bc}$ & $152 \mathrm{Aa}$ & $134 \mathrm{Ac}$ & 08Bc & $42 \mathrm{Bb}$ & $\mathrm{Aa}$ & $1,99 \mathrm{Aj}$ & $1,85 \mathrm{Ad}$ & $20 \mathrm{Ab}$ & $62,9 \mathrm{Bc}$ & $9 \mathrm{Aa}$ & $186 \mathrm{Ab}$ & 88Aa & $\mathrm{Ab}$ & $1,05 \mathrm{~b}$ \\
\hline ouradão & $35,7 \mathrm{Ba}$ & $39,4 \mathrm{Aa}$ & $122 \mathrm{Bd}$ & $137 \mathrm{Ac}$ & $127 \mathrm{Ac}$ & $103 \mathrm{Bc}$ & $15 \mathrm{Be}$ & $40 \mathrm{Ac}$ & $3,01 \mathrm{Ae}$ & $2,78 \mathrm{Ab}$ & 26 & $140 \mathrm{Bc}$ & $\mathrm{Aa}$ & $191 \mathrm{Ab}$ & 20 & 149Aa & $0,69 b$ \\
\hline roz &, $6 \mathrm{Ba}$ & $9,1 \mathrm{Aa}$ & $149 \mathrm{Ab}$ & $156 \mathrm{Aa}$ & $139 \mathrm{Ab}$ & $115 \mathrm{I}$ & $50 \mathrm{I}$ & a & $2,06 \mathrm{Aj}$ & $1,89 \mathrm{Ad}$ & $142 \mathrm{Ac}$ & $2,83 \mathrm{Bc}$ & $\mathrm{Aa}$ & $186 \mathrm{Ab}$ & Aa & $\mathrm{b}$ & $5 a$ \\
\hline roz & $37,2 \mathrm{Aa}$ & $39,1 \mathrm{Aa}$ & $110 \mathrm{Bf}$ & $24 \mathrm{Ad}$ & $115 \mathrm{Ad}$ & $86 \mathrm{E}$ & 36 & a & $2,39 \mathrm{Bh}$ & $2,7 \mathrm{Ab}$ & $\mathrm{Ab}$ & Bc & $\mathrm{Aa}$ & $\mathrm{Aa}$ & Aa & $\mathrm{a}$ & $5 a$ \\
\hline aponês & $35 \mathrm{Ba}$ & $\mathrm{Aa}$ & $111 \mathrm{Bf}$ & $134 \mathrm{Ac}$ & $93 \mathrm{Ae}$ & & $15 \mathrm{Be}$ & $53 \mathrm{Ab}$ & $2,23 \mathrm{Ai}$ & $1,75 \mathrm{Bd}$ & 34 & Bc & $\mathrm{Aa}$ & 178 & $\mathrm{Aa}$ & $\mathrm{Ab}$ & $1,31 \mathrm{a}$ \\
\hline gul & $35,1 \mathrm{Ba}$ & $37,6 \mathrm{Aa}$ & $110 \mathrm{Bf}$ & $125 \mathrm{Ad}$ & $33 \mathrm{Ac}$ & & 28 & & $3,37 \mathrm{Ad}$ & & b & $3 c$ & Aa & $\mathrm{a}$ & ta & a & $5 \mathrm{~b}$ \\
\hline rroz & $34,1 \mathrm{Ba}$ &, $2 \mathrm{Aa}$ & $124 \mathrm{Bd}$ & $138 \mathrm{Ac}$ & $123 \mathrm{Ac}$ & 101 & 22 & $\mathrm{c}$ & $2,62 \mathrm{Ag}$ & $2,34 \mathrm{Ac}$ & $312 \mathrm{Aa}$ & $\mathrm{Bc}$ & $\mathrm{Aa}$ & 182 & $\mathrm{Aa}$ & $b$ & $1,03 a$ \\
\hline rro & $34,4 \mathrm{Aa}$ & $6,8 \mathrm{Aa}$ & $148 \mathrm{Ab}$ & $151 \mathrm{Aa}$ & $145 \mathrm{Ab}$ & $113 \mathrm{Bc}$ & $46 \mathrm{Bb}$ & $\mathrm{Aa}$ & Bl & $1,69 \mathrm{Ad}$ & $74,3 \mathrm{Ac}$ & $\mathrm{Ac}$ & $\mathrm{Aa}$ & $\mathrm{db}$ & 18Aa & $8 b$ & $0,95 \mathrm{~b}$ \\
\hline rro & $34,8 \mathrm{Ba}$ & 8,6Aa & $117 \mathrm{Be}$ & $141 \mathrm{Ac}$ & $127 \mathrm{Ac}$ & $116 \mathrm{Ab}$ & $26 \mathrm{Bd}$ & 52 & $2,76 \mathrm{Ag}$ & $2,3 \mathrm{Bc}$ & 26 & $68,6 \mathrm{Bc}$ & $\mathrm{Aa}$ & 15 & 19 & Bb & 1a \\
\hline exo & 9Aa & $6,7 \mathrm{Aa}$ & $129 \mathrm{Ad}$ & $137 \mathrm{Ac}$ & $122 \mathrm{Ac}$ & 104 & 30 & 5 & $2,2 \mathrm{Ai}$ & $1,79 \mathrm{Bd}$ & $269 \mathrm{Ab}$ & $\mathrm{Bb}$ & $4 \mathrm{a}$ & 16 & $\mathrm{Aa}$ & b & $0,82 \mathrm{~b}$ \\
\hline 4 Dias & $36,2 \mathrm{Aa}$ & 7,6Aa & $67 \mathrm{Ak}$ & 76Ah & $97 \mathrm{Ae}$ & & $28 \mathrm{Bd}$ & $\mathrm{Aa}$ & $3,52 \mathrm{Ac}$ & $2,95 \mathrm{Ba}$ & $320 \mathrm{Aa}$ & $52,4 \mathrm{Bc}$ & $273 \mathrm{Aa}$ & $209 \mathrm{Aa}$ & $235 \mathrm{Aa}$ & 17 & $1,23 \mathrm{a}$ \\
\hline ambari & $35 \mathrm{Aa}$ & $1 \mathrm{Aa}$ & $144 \mathrm{Ab}$ & $152 \mathrm{Aa}$ & $149 \mathrm{Aa}$ & $111 \mathrm{Bc}$ & $55 \mathrm{Bb}$ & $92 \mathrm{Aa}$ & $1,68 \mathrm{Ak}$ & $\mathrm{Ad}$ & $144 \mathrm{Ac}$ & $37,9 \mathrm{Ac}$ & $20 \mathrm{Aa}$ & $179 \mathrm{Bb}$ & 79Aa & $38 \mathrm{Bb}$ & $1,09 \mathrm{a}$ \\
\hline enith & $37,1 \mathrm{Aa}$ & $6 \mathrm{Aa}$ & $115 \mathrm{Ae}$ & $123 \mathrm{Ae}$ & $110 \mathrm{Ad}$ & $3 \mathrm{~d}$ & $24 \mathrm{Bd}$ & $78 \mathrm{Aa}$ & $2,28 \mathrm{Ai}$ & $1,71 \mathrm{Bd}$ & $360 \mathrm{Aa}$ & $12,1 \mathrm{Bc}$ & $\mathrm{Aa}$ & $248 \mathrm{Aa}$ & $\mathrm{Aa}$ & 3a & $1,43 a$ \\
\hline edad & ,3Ba & ,7Aa & $133 \mathrm{Ac}$ & $139 \mathrm{Ac}$ & $129 \mathrm{Ac}$ & $8 \mathrm{E}$ & 391 & $\mathrm{a}$ & $2,29 \mathrm{Ai}$ & $1,87 \mathrm{Bd}$ & $248 \mathrm{Ab}$ & $52,4 \mathrm{Bc}$ & $211 \mathrm{Aa}$ & $156 \mathrm{Ab}$ & $192 \mathrm{Aa}$ & 20Bb & $1,16 \mathrm{a}$ \\
\hline gull & $5,5 \mathrm{Ba}$ & $9,1 \mathrm{Aa}$ & $150 \mathrm{Bb}$ & $159 \mathrm{Aa}$ & $155 \mathrm{Aa}$ & $122 \mathrm{Bb}$ & $50 \mathrm{Ab}$ & $65 \mathrm{Aa}$ & $2,03 \mathrm{Aj}$ & $2,01 \mathrm{Ac}$ & $153 \mathrm{Ac}$ & $31,4 \mathrm{Bc}$ & $92 \mathrm{Aa}$ & $199 \mathrm{Bb}$ & $276 \mathrm{Aa}$ & $170 \mathrm{Ba}$ & $1,17 \mathrm{a}$ \\
\hline ia & - & 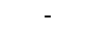 & - & 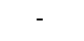 & 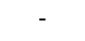 & 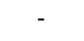 & 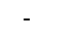 & 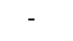 & - & 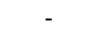 & & & 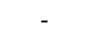 & & - & 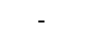 & - \\
\hline atoba &, $5 \mathrm{Ba}$ &, $3 \mathrm{Aa}$ & $123 \mathrm{Bd}$ & $133 \mathrm{Ac}$ & $127 \mathrm{Ac}$ & 11 & 23 & $32 \mathrm{Ac}$ & 3,39Ad & 3,1 & $367 \mathrm{Aa}$ & $\mathrm{Ba}$ & Аa & $192 \mathrm{Ab}$ & 209Aa & $153 \mathrm{Aa}$ & $0,56 \mathrm{~b}$ \\
\hline $\mathrm{rac}$ & $4 \mathrm{Ba}$ & 8,9Aa & $147 \mathrm{Bb}$ & $159 \mathrm{Aa}$ & $146 \mathrm{Ab}$ & $119 \mathrm{Bb}$ & $56 \mathrm{I}$ & $94 \mathrm{Aa}$ & $1,74 \mathrm{Ak}$ & $1,55 \mathrm{Ad}$ & $134 \mathrm{Ac}$ & $5,23 \mathrm{Bc}$ & 319Aa & $229 \mathrm{Ba}$ & $252 \mathrm{Aa}$ & $192 \mathrm{Aa}$ & $1,42 \mathrm{a}$ \\
\hline urit & $34,4 \mathrm{Ba}$ & 7,7Aa & $124 \mathrm{Bd}$ & $134 \mathrm{Ac}$ & $104 \mathrm{Ad}$ & $111 \mathrm{Ac}$ & $25 \mathrm{Bd}$ & $57 \mathrm{Ab}$ & Af & & $300 \mathrm{Aa}$ & $92,9 \mathrm{Bc}$ & $\mathrm{Aa}$ & & $4 a$ & $\mathrm{Bb}$ & $1,02 \mathrm{a}$ \\
\hline Гарuripa & $34,8 \mathrm{Ba}$ & $38,5 \mathrm{Aa}$ & $156 \mathrm{Aa}$ & $160 \mathrm{Aa}$ & $102 \mathrm{Ad}$ & 83 & 89Aa & 89Aa & $2,23 \mathrm{Ai}$ & $1,65 \mathrm{Bd}$ & $44,3 \mathrm{Ac}$ & $5,23 \mathrm{Ac}$ & $375 \mathrm{Aa}$ & $233 \mathrm{Ba}$ & 39Aa & $117 \mathrm{Bb}$ & $1,3 \mathrm{a}$ \\
\hline $\mathrm{RS}$ & ,2Ba & $39 \mathrm{Aa}$ & $97 \mathrm{Bh}$ & $122 \mathrm{Ae}$ & $90 \mathrm{Ae}$ & & 20 & $58 \mathrm{Ab}$ & $2,67 \mathrm{Ag}$ & $2,22 \mathrm{Bc}$ & $464 \mathrm{Aa}$ & $127 \mathrm{Bb}$ & $278 \mathrm{Aa}$ & $232 \mathrm{Aa}$ & $232 \mathrm{Aa}$ & $173 \mathrm{Aa}$ & $1,07 \mathrm{a}$ \\
\hline BRS &, $5 \mathrm{Ba}$ & $38,1 \mathrm{Aa}$ & 89Bi & $105 \mathrm{Ag}$ & $102 \mathrm{Ad}$ & 89Ad & $42 \mathrm{Ab}$ & $52 \mathrm{Ab}$ & 2,5Ah & $2,16 \mathrm{Bc}$ & $230 \mathrm{Ab}$ & $93,8 \mathrm{Bc}$ & $244 \mathrm{Aa}$ & $172 \mathrm{Ab}$ & $178 \mathrm{Aa}$ & $132 \mathrm{Ab}$ & $0,87 \mathrm{~b}$ \\
\hline BRS Monarca & $35,5 \mathrm{Aa}$ & $37,5 \mathrm{Aa}$ & 86Ai & $94 \mathrm{Ag}$ & $87 \mathrm{Ae}$ & $74 \mathrm{Ae}$ & $31 \mathrm{Ac}$ & $28 \mathrm{Ac}$ & $3,28 \mathrm{Ad}$ & $2,76 \mathrm{Bb}$ & $364 \mathrm{Aa}$ & $129 \mathrm{Bb}$ & $305 \mathrm{Aa}$ & $242 \mathrm{Aa}$ & $269 \mathrm{Aa}$ & $173 \mathrm{Ba}$ & $0,95 \mathrm{~b}$ \\
\hline BRS & ,1 Aa & $38,5 \mathrm{Aa}$ & $91 \mathrm{Bh}$ & $105 \mathrm{Ag}$ & $93 \mathrm{Ae}$ & $68 \mathrm{Be}$ & $28 \mathrm{Bd}$ & $63 \mathrm{Aa}$ & $3,26 \mathrm{Ad}$ & $2,34 \mathrm{Bc}$ & $449 \mathrm{Aa}$ & $45 \mathrm{Bc}$ & $286 \mathrm{Aa}$ & $216 \mathrm{Aa}$ & $253 \mathrm{Aa}$ & $180 \mathrm{Ba}$ & $1,33 \mathrm{a}$ \\
\hline ico & $34,5 \mathrm{Ba}$ & $39,9 \mathrm{Aa}$ & $125 \mathrm{Bd}$ & $142 \mathrm{Ac}$ & $133 \mathrm{Ac}$ & 1 & 30 & $47 \mathrm{Ab}$ & $3 \mathrm{Ae}$ & $2,62 \mathrm{Bb}$ & $293 \mathrm{Aa}$ & $161 \mathrm{Bb}$ & $235 \mathrm{Aa}$ & $166 \mathrm{Ab}$ & $198 \mathrm{Aa}$ & $127 \mathrm{Bb}$ & $0,67 \mathrm{~b}$ \\
\hline BRS Primavera & $34,8 \mathrm{Ba}$ & $40,4 \mathrm{Aa}$ & 97Bh & $117 \mathrm{Ae}$ & $114 \mathrm{Ad}$ & & $53 \mathrm{Bb}$ & $89 \mathrm{Aa}$ & $2,27 \mathrm{Ai}$ & $2,25 \mathrm{Ac}$ & $\mathrm{Ab}$ & $16,7 \mathrm{Bc}$ & $\mathrm{Aa}$ & $\mathrm{Aa}$ & $33 \mathrm{Aa}$ & $97 \mathrm{Aa}$ & $1,37 \mathrm{a}$ \\
\hline horinho & $33,1 \mathrm{Ba}$ & $40 \mathrm{Aa}$ & $122 \mathrm{Bd}$ & $138 \mathrm{Ac}$ & $128 \mathrm{Ac}$ & $114 \mathrm{Ab}$ & $24 \mathrm{Bd}$ & $48 \mathrm{Ab}$ & $2,61 \mathrm{Ag}$ & $2,67 \mathrm{Ab}$ & $307 \mathrm{Aa}$ & $150 \mathrm{Bb}$ & $187 \mathrm{Aa}$ & $141 \mathrm{Ab}$ & $158 \mathrm{Aa}$ & $106 \mathrm{Ab}$ & $0,76 \mathrm{~b}$ \\
\hline 2601 & $5 \mathrm{Ba}$ & $38,9 \mathrm{Aa}$ & $89 \mathrm{Bi}$ & $101 \mathrm{Ag}$ & 78Ae & & $24 \mathrm{Bd}$ & $71 \mathrm{Aa}$ & $2,7 \mathrm{Ag}$ & $2,08 \mathrm{Bc}$ & $324 \mathrm{Aa}$ & $103 \mathrm{Bc}$ & 336Aa & $244 \mathrm{Ba}$ & $291 \mathrm{Aa}$ & $193 \mathrm{Ba}$ & $1,01 \mathrm{~b}$ \\
\hline & $3 \mathrm{Ba}$ & $39,5 \mathrm{Aa}$ & $97 \mathrm{Bh}$ & $114 \mathrm{Af}$ & $101 \mathrm{Ad}$ & & 31 & $74 \mathrm{Aa}$ & $2,53 \mathrm{Ah}$ & $2,4 \mathrm{Ab}$ & $253 \mathrm{Ab}$ & $38,6 \mathrm{Bc}$ & $193 \mathrm{Aa}$ & & 63Аа & $50 \mathrm{Ad}$ & $1,25 \mathrm{a}$ \\
\hline 535 & $3,5 \mathrm{Ba}$ & $38 \mathrm{Aa}$ & $115 \mathrm{Be}$ & $125 \mathrm{Ad}$ & $119 \mathrm{Ac}$ & 88 & $20 \mathrm{Bd}$ & $56 \mathrm{Ab}$ & $2,22 \mathrm{Ai}$ & $9 \mathrm{Bd}$ & $553 \mathrm{Aa}$ & $121 \mathrm{Bb}$ & 00Aa & 10Ba & $12 \mathrm{Aa}$ & $4 \mathrm{Ba}$ & $1,15 \mathrm{a}$ \\
\hline Ens & $33,2 \mathrm{Ba}$ & $39,5 \mathrm{Aa}$ & $98 \mathrm{Bh}$ & $123 \mathrm{Ae}$ & 109Ad & & $33 \mathrm{Bc}$ & $83 \mathrm{Aa}$ & 2,5Ah & $2,03 \mathrm{Bc}$ & $337 \mathrm{Aa}$ & $25,2 \mathrm{Bc}$ & $254 \mathrm{Aa}$ & $204 \mathrm{Aa}$ & $212 \mathrm{Aa}$ & $145 \mathrm{Ab}$ & $1,37 \mathrm{a}$ \\
\hline & & & 06Bg & & & & & & & & & & & & & 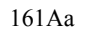 & $0,69 \mathrm{~b}$ \\
\hline 051 & Ba & $9,2 \mathrm{Aa}$ & $102 \mathrm{Bg}$ & $123 \mathrm{Ae}$ & 108Ad & & 42 & 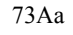 & $6 \mathrm{~A}$ & $2,11 \mathrm{Bc}$ & $222 \mathrm{~A}$ & $\mathrm{Bc}$ & $269 \mathrm{Aa}$ & $211 \mathrm{Aa}$ & $208 \mathrm{Aa}$ & $155 \mathrm{Aa}$ & $1,07 \mathrm{a}$ \\
\hline ס & $34,3 \mathrm{Ba}$ & $38,3 \mathrm{Aa}$ & $94 \mathrm{Bh}$ & 109Af & $95 \mathrm{Ae}$ & $75 \mathrm{Be}$ & $27 \mathrm{Bd}$ & $79 \mathrm{Aa}$ & $2,92 \mathrm{Af}$ & $2,41 \mathrm{Bb}$ & $362 \mathrm{Aa}$ & $52,9 \mathrm{Bc}$ & $268 \mathrm{Aa}$ & $235 \mathrm{Aa}$ & 239Aa & $198 \mathrm{Aa}$ & $1,26 \mathrm{a}$ \\
\hline
\end{tabular}

${ }^{(1)}$ Médias seguidas de letras iguais, maiúsculas nas comparações entre as condições com e sem estresse, não diferem significativamente pelo teste de Tukey, a $5 \%$ de probabilidade; enquanto médias seguidas de letras iguais, minúsculas, fazem parte do mesmo grupo pelo teste de Scott-Knott, a $5 \%$ de probabilidade. ${ }^{(2)}$ Rabo de Carneiro. ${ }^{(3)}$ BGCR, Bico Ganga Cana Roxa. ${ }^{(4)} \mathrm{BCD}$, Beira Campo Dourado. 
Observou-se correlação positiva significativa entre FLO e ALT, nos dois ambientes de cultivo (Tabela 3), indício de que os genótipos com maior ciclo tenderam a apresentar maior altura. No ambiente sem estresse hídrico, a FLO correlacionou-se negativamente com a produtividade de grãos $(\mathrm{r}=-0,3310)$, o que indica que genótipos mais precoces tenderam a ser mais produtivos nesse ambiente. Entretanto, não houve correlação significativa entre essas variáveis no ambiente com estresse, no presente trabalho. Pantuwan et al. (2002), Bernier et al. (2007) e Kumar et al. (2008), ao estudarem o efeito do estresse hídrico em linhagens de arroz, encontraram correlação negativa entre produtividade e FLO, o que é indicativo de que genótipos mais precoces apresentam mecanismo de escape, com aceleração do desenvolvimento sob condições moderadas de estresse.

A altura dos genótipos sofreu influência dos ambientes de cultivo (Tabela 1), tendo-se verificado tanto genótipos de porte bem baixo, como o Chorinho (com 77 e $58 \mathrm{~cm}$, nas condições sem e com estresse, respectivamente), quanto de porte alto, como o Brejeiro/ Nenenzinho (com 156 e $138 \mathrm{~cm}$, respectivamente). A altura diminuiu no ambiente com estresse de seca, em todos os genótipos avaliados (Tabela 2), com redução média de $21 \mathrm{~cm}$; alguns materiais, como os genótipos Lambari e Enche Tulha, apresentaram drástica diminuição na variável, de cerca de $38 \mathrm{~cm}$.

A EST é considerada um dos componentes primários da produção de grãos em arroz (Alvarez et al., 2012). Os genótipos avaliados apresentaram diferenças altamente significativas quanto a essa variável, que, por sua vez, apresentou forte interação com as condições de cultivo (Tabela 1). A esterilidade média foi de 28,34 e $58,72 \%$, respectivamente, para os ambientes sem e com estresse hídrico (Tabela 2). Observou-se grande variação nos valores de esterilidade entre os genótipos mesmo no ambiente sem estresse, como os registrados para Tapuripa e Formosa, que apresentaram EST, nesse ambiente, de 89 e $6 \%$, respectivamente. No ambiente com estresse, os valores de EST aumentaram, em média, 30,38\%, e alguns genótipos aproximaram-se da esterilidade total, como o Caiana Grande, com 95\% das espiguetas estéreis (Tabela 2).

Apesar da grande variabilidade em EST na coleção nuclear, esse resultado deve ser considerado com cautela, já que outras variaveis meteorológicas, como baixa umidade relativa do ar e elevadas temperaturas, podem contribuir para seu aumento. Elevadas temperaturas diurnas podem aumentar o percentual de esterilidade das espiguetas mesmo sob irrigação normal (Jagadish et al., 2007). Ainda assim, essa variável tem grande potencial de uso na seleção de linhagens de arroz superiores quanto à tolerância à seca (Jongdee et al., 2006).

A coincidência do estresse hídrico com a meiose das células dos grãos de pólen é o principal fator para esterilidade das espiguetas (Jin et al., 2013).

Foram observadas correlações negativas entre EST e produtividade de grãos, tanto na condição com estresse quanto na sem estresse hídrico $(r=-0,8812$ e $r=-0,7072$, respectivamente).

O P100 variou significativamente entre os genótipos e entre as condições de cultivo (Tabela 1). Na ausência

Tabela 3. Estimativas das correlações entre as características avaliadas, nos ambientes com (diagonal superior) e sem (diagonal inferior) estresse hídrico ${ }^{(1)}$.

\begin{tabular}{lccccccccc}
\hline & TDF & FLO & ALT & PRO & EST & P100 & PER & PAN \\
\hline TDF & - & $-0,012$ & $-0,114$ & 0,078 & $-0,032$ & $-0,006$ & 0,030 & $-0,164$ \\
FLO & $-0,159$ & - & $0,711^{* *}$ & 0,140 & $-0,011$ & $-0,441^{* *}$ & $-0,498^{* *}$ & $-0,509^{* *}$ & $-0,145$ \\
ALT & $-0,106$ & $0,800^{* *}$ & - & $0,331^{*}$ & $-0,175$ & 0,023 & $-0,767^{* *}$ & $-0,753^{* *}$ & $-0,56^{* *}$ \\
PRO & $-0,264$ & $-0,331^{*}$ & $-0,332^{*}$ & - & $-0,881^{* *}$ & $0,480^{* *}$ & $-0,639^{* *}$ & $-0,613^{* *}$ & $-0,8114^{* *}$ \\
EST & 0,226 & 0,22 & 0,164 & $-0,703^{* *}$ & - & $-0,602 * *$ & $0,700^{* *}$ & $0,615^{* *}$ & $0,814^{* *}$ \\
P100 & $-0,034$ & $-0,627 * *$ & $-0,386^{*}$ & $0,395^{*}$ & $-0,459^{* *}$ & - & $-0,417^{* *}$ & $-0,402^{* *}$ & $-0,324^{*}$ \\
PER & 0,029 & $-0,278$ & $-0,629^{* *}$ & $-0,139$ & 0,243 & 0,069 & - & $0,936^{* *}$ & $0,671^{* *}$ \\
PAN & 0,056 & $-0,353^{*}$ & $-0,625^{* *}$ & $-0,145$ & $0,406^{* *}$ & $-0,021$ & $0,884^{* *}$ & - \\
ISS & 0,179 & $-0,239$ & $-0,350^{*}$ & $-0,128$ & $0,400^{* *}$ & $-0,123$ & $0,620^{* *}$ & $0,779^{* *}$ & $0,682^{* *}$ \\
\hline
\end{tabular}

${ }^{(1)} \mathrm{TDF}$, temperatura do dossel foliar; FLO, número de dias da emergência à floração média; ALT, altura de planta; PRO, produtividade de grãos; EST, percentagem de esterilidade de espiguetas; P100, peso de 100 grãos; PER, número de perfilhos por metro; PAN, número de panículas por metro; e ISS, índice de suscetibilidade à seca. ${ }^{*} \mathrm{e} *$ Significativo pelo teste $\mathrm{t}$, a 5 e $1 \%$ de probabilidade, respectivamente. 
do estresse hídrico, foram observados desde genótipos com grãos maiores e mais pesados, como o Beira Campo Dourado $(3,95 \mathrm{~g})$, até materiais com grãos menores e mais leves, como o Arroz Agulha (1,31 g) (Tabela 2). No ambiente com estresse hídrico, o P100 diminuiu, em média, 0,43 g. Porém, mesmo nessas condições, alguns genótipos mantiveram elevado P100, como o Santo Américo (3,22 g) (Tabela 2). A redução no peso dos grãos, na condição de estresse, é atribuída à menor produção e exportação de carboidratos para o enchimento dos grãos (Jagadish et al., 2007).

$\mathrm{Na}$ condição sem estresse, o P100 correlacionouse positivamente com a produtividade de grãos, e negativamente com EST, PER, PAN e ISS (Tabela 3). Na condição com estresse, o P100 correlacionou-se positivamente com a produtividade, e negativamente com FLO, ALT e EST.

No ambiente sem estresse hídrico, os genótipos não diferiram significativamente quanto às variáveis PER e PAN. Já no ambiente com estresse, houve diferença significativa entre os genótipos (Tabela 1). Verificouse tendência de diminuição no número de perfilhos e panículas, em condições de seca. Nessas condições, entre os genótipos com maiores valores para essas variáveis, destaca-se o Carioca/Rabo de Carneiro, com PER de 275 e PAN de 207 (Tabela 2). Os genótipos Enche Tulha e Arroz Catetão foram os que, em condições de estresse, apresentaram o menor número de perfilhos e panículas, 138 e 96, respectivamente. $\mathrm{O}$ índice médio de perfilhos estéreis no ambiente com estresse foi de $33 \%$. Esse resultado reforça a tese de que cultivares de arroz de sequeiro, para que possam tolerar estresses hídricos, devem apresentar menor número de perfilhos por planta, que sejam, na grande maioria, férteis.

As variáveis PER e PAN apresentaram correlações negativas e altamente significativas $(\mathrm{r}=-0,6393$ e $\mathrm{r}=-0,6126$, respectivamente) com a produtividade de grãos no ambiente com estresse, indício de que os genótipos mais produtivos tendem a ter menor número de perfilhos, mas maior prevalência de perfilhos férteis. No ambiente sem estresse hídrico, essas variáveis não se correlacionaram significativamente com a produtividade.

Foi observada ampla variação nos valores de produtividade de grãos, com diferenças altamente significativas entre os genótipos avaliados (Tabela 1). $\mathrm{Na}$ ausência de estresse hídrico, o genótipo Arroz Carolino destacou-se como o mais produtivo, com
$626,66 \mathrm{~g}$ por parcela, enquanto o menos produtivo foi o Tapuripa, com $62 \mathrm{~g}$ (Tabela 2). Na presença do estresse, o Catetão, com $352 \mathrm{~g}$ foi o mais produtivo, e o Agulhinha Tardio, com 2,67 g, o menos produtivo.

A produtividade de grãos diminuiu $68 \%$ entre os ambientes sem (391,72 g) e com estresse hídrico (126,35 g) (Tabela 2). Entretanto, alguns genótipos, como Ligeiro, Canela de Ferro, Arroz do Sul e Muruim Branco1, apesar da baixa produtividade em ambas as condições, apresentaram pequena redução com o estresse hídrico. Os genótipos Catetão, Jatobá, Arroz Piriquito, Arroz Maranhão, Muruim Branco2, Bico Ganga Cana Roxa, Chorinho, Bico Ganga Curto e Puteca apresentaram pequena redução na produtividade e elevada produtividade nos dois ambientes. Portanto, é provável que esses dois grupos de genótipos apresentem alelos favoráveis à tolerância à seca, que podem ser úteis nos programas de melhoramento do arroz de sequeiro.

A maioria das cultivares comerciais (BRS Curinga, BRS Pepita, BRS Monarca, BRS Sertaneja e BRS Primavera) e das linhagens elites (BRA02601, BRA01506, BRA02535, BRA042156, BRA042160, BRA032051 e BRA032033), oriundas do programa de melhoramento genético da Embrapa Arroz e Feijão, apresentou elevada produtividade de grãos na condição sem estresse hídrico; porém, houve acentuada redução nessa variável na condição com estresse. Esse resultado evidencia perda da tolerância à seca nos materiais procedentes desse programa de melhoramento.

A ampla diversidade genética entre os acessos da coleção nuclear fez-se notar, também, em diferenças altamente significativas nos valores de ISS, entre os genótipos (Tabela 1). Foram observados desde genótipos com baixo ISS, como o Ligeiro $(0,15)$, considerado tolerante à seca, até genótipos com ISS bastante elevado, como o Agulhinha Tardio $(1,459)$, considerado susceptível à seca. Os acessos Ligeiro e Canela de Ferro foram os que apresentaram o menor ISS, de 0,15 e 0,25, respectivamente. Apesar das baixas produtividades de grãos alcançadas por esses genótipos mesmo no ambiente sem estresse (Tabela 2), eles podem ser importantes como fonte de alelos favoráveis em genes candidatos para tolerância à seca.

Constataram-se correlações significativas do ISS com todas as demais características avaliadas, no ambiente sob estresse hídrico, com exceção da TDF (Tabela 3). Esse resultado corrobora a importância desse parâmetro na seleção de genótipos tolerantes 
à seca. No ambiente sem estresse hídrico, a altura de planta, a esterilidade de espiguetas e o número de perfilhos e panículas apresentaram correlações significativas com essa variável.

Os longos anos de cultivo, em condições de sequeiro, das variedades tradicionais de arroz no Brasil, muitas delas introduzidas na época do descobrimento do País (Rangel et al., 2013), levaram a sua adaptação a este ambiente, com acúmulo de alelos que as permitem suportar esse estresse hídrico.

\section{Conclusões}

1. A temperatura do dossel foliar tem pouca importância na seleção de genótipos de arroz de terras altas (Oryza sativa) tolerantes ou sensíveis à seca.

2. A esterilidade das espiguetas é uma variável importante na seleção de plantas de arroz de terras altas produtivas, tanto na condição sem estresse quanto na com estresse hídrico, em razão de sua elevada correlação negativa com a produtividade.

3. O índice de susceptibilidade à seca correlacionase significativamente com diversas características de interesse na cultura do arroz de terras altas submetida ao estresse hídrico e, portanto, trata-se de um importante meio para a seleção de plantas nessas condições.

\section{Agradecimentos}

À Coordenação de Aperfeiçoamento de Pessoal de Nível Superior (Capes) e à Embrapa, pela concessão de bolsa.

\section{Referências}

ALVAREZ, R. de C.F.; CRUSCIOL, C.A.C.; NASCENTE, A.S.; RODRIGUES, J.D.; HABERMANN, G. Gas exchange rates, plant height, yield components, and productivity of upland rice as affected by plant regulators. Pesquisa Agropecuária Brasileira, v.47, p.1455-1461, 2012. DOI: 10.1590/S0100-204X2012001000007.

BABU, R.C.; NGUYEN, B.D.; CHAMARERK, V.; SHANMUGASUNDARAM,P.; CHEZHIAN,P.; JEYAPRAKASH, P.; GANESH, S.K.; PALCHAMY, A.; SADASIVAM, S.; SARKARUNG, S.; WADE, L.J.; NGUYEN, H.T. Genetic analysis of drought resistance in rice by molecular markers: association between secondary traits and field performance. Crop Science, v.43, p.1457-1469, 2003. DOI: 10.2135/cropsci2003.1457.

BERNIER, J.; KUMAR, A.; RAMAIAH, V.; SPANER, D.; ATLIN, G. A large-effect QTL for grain yield under reproductive-stage drought stress in upland rice. Crop Science, v.47, p.507-518, 2007. DOI: $10.2135 /$ cropsci2006.07.0495.
CENTENO, A.J. Curso de estatística aplicada à biologia. 2.ed. Goiânia: Ed. da UFG, 2001. 234p.

FISCHER, R.A.; MAURER, R. Drought resistance in spring wheat cultivars. I. Grain yield responses. Australian Journal of Agricultural Research, v.29, p.897-912, 1978. DOI: 10.1071/ AR9780897.

HUANG, Y.M.; XIAO, B.Z.; XIONG, L.Z. Characterization of a stress responsive proteinase inhibitor gene with positive effect in improving drought resistance in rice. Planta, v.226, p.73-85, 2007. DOI: 10.1007/s00425-006-0469-8.

JAGADISH, S.V.K.; CRAUFURD, P.Q.; WHEELER, T.R. High temperature stress and spikelet fertility in rice (Oryza sativa L.). Journal of Experimental Botany, v.58, p.1627-1635, 2007. DOI: 10.1093/jxb/erm003.

JIN, Y.; YANG, H.X.; WEI, Z.; MA, H.; GE, X.C. Rice male development under drought stress: phenotypic changes and stage-dependent transcriptomic reprogramming. Molecular Plant, v.6, p.1630-1645, 2013. DOI: 10.1093/mp/sst067.

JONGDEE, B.; PANTUWAN, G.; FUKAI, S.; FISCHER, K. Improving drought tolerance in rainfed lowland rice: an example from Thailand. Agricultural Water Management, v.80, p.225-240, 2006. DOI: 10.1016/j.agwat.2005.07.015.

KUMAR, A.; BERNIER, J.; VERULKAR, S.; LAFITTE, H.R.; ATLIN, G.N. Breeding for drought tolerance: direct selection for yield, response to selection and use of drought-tolerant donors in upland and lowland-adapted populations. Field Crops Research, v.107, p.221-231, 2008. DOI:10.1016/j.fcr.2008.02.007.

NGUYEN, H.T.; BABU, R.C.; BLUM, A. Breeding for drought resistance in rice: physiology and molecular genetics considerations. Crop Science, v.37, p.1426-1437, 1997. DOI: 10.2135/cropsci199 7.0011183X003700050002x.

PANTUWAN, G.; FUKAI, S.; COOPER, M.; RAJATESEREEKUL, S.; O'TOOLE, J.C. Yield response of rice (Oryza sativa L.) genotypes to drought under rainfed lowlands 2. Selection of drought resistant genotypes. Field Crops Research, v.73, p.169-180, 2002. DOI: 10.1016/S0378-4290(01)00195-2.

PESSOA-FILHO, M.; BELÓ, A.; ALCOCHETE, A.A.N.; RANGEL, P.H.N.; FERREIRA, M.E. A set of multiplex panels of microsatellite markers for rapid molecular characterization of rice accessions. BMC Plant Biology, v.7, article 23, 2007. DOI: 10.1186/1471-2229-7-23.

RANGEL, P.H.N.; OLIVEIRA, J.P. de; COSTA, J.G.C.; FERREIRA, M.E.; ABREU, A.G. de. Banco ativo de germoplasma de arroz e feijão: passado, presente e futuro. Santo Antônio de Goiás. Embrapa Arroz e Feijão, 2013. 68p. (Embrapa Arroz e Feijão. Documentos, 288).

SHINOZAKI, K.; YAMAGUCHI-SHINOZAKI, K. Gene networks involved in drought stress response and tolerance. Journal of Experimental Botany, v.58, p.221-227, 2007. DOI: 10.1093/jxb/ erl164.

STONE, L.F.; MOREIRA, J.A.A.; SILVA, S.C. da. Tensão da água do solo e produtividade do arroz. Goiânia: EMBRAPA-CNPAF, 1986. 6p. (EMBRAPA-CNPAF. Comunicado técnico, 19). 
UGA, Y.; SUGIMOTO, K.; OGAWA, S.; RANE, J.; ISHITANI, M.; HARA, N.; KITOMI, Y.; INUKAI, Y.; ONO, K.; KANNO, N.; INOUE, H.; TAKEHISA, H.; MOTOYAMA, R.; NAGAMURA, Y.; WU, J.Z.; MATSUMOTO, T.; TAKAI, T.; OKUNO, K.; YANO, M. Control of root system architecture by DEEPER ROOTING 1 increases rice yield under drought conditions. Nature Genetics, v.45, p.1097-1102, 2013. DOI: 10.1038/ng.2725.
VIDAL, M.S.; CARVALHO, J.M.F.C.; MENESES, C.H.S.G. Déficit hídrico: aspectos morfofisiológicos. Campina Grande: Embrapa Algodão, 2005. 19p. (Embrapa Algodão. Documentos, 142).

XU, Y.; MCCOUCH, S.R.; ZHANG, Q. How can we use genomics to improve cereals with rice as a reference genome? Plant Molecular Biology, v.59, p.7-26, 2005. DOI: 10.1007/ s11103-004-4681-2.

Recebido em 2 de fevereiro de 2015 e aprovado em 29 de junho de 2015 